\title{
LAS ABEJAS NATIVAS, MELLIPONAS (HYMENOPTERA: MELLIPONIDAE) ASOCIADAS AL CAMU CAMU Myrciaria dubia
}

\author{
Cesar DELGADO ${ }^{1}$, Marllori VELA ${ }^{1}$ \\ 1 Instituto de Investigaciones de la Amazonía Peruana. Av. A. Quiñones Km. 2,5. Iquitos - Perú. Apartado 784, \\ cdelgado@iiap.org.pe
}

\section{RESUMEN}

De abril de 2005 a febrero del 2006, fueron muestreadas las inflorescencias de camu camu en cinco comunidades del bajo rio Amazonas, con el objetivo de conocer las especies y abundancia de abejas nativas asociadas a esta especie vegetal. Se registraron 6 géneros y 16 especies de abejas nativas y Aphis mellifera como especie introducida. Las mayores riquezas de especies fueron presentadas por los géneros Trigona con $43.75 \%$ y Melipona con $25 \%$. Las especies más abundantes fueron Trigona sp. 1 con $42.40 \%$ y Melipona eburnea con $22.12 \%$. Se realiza una breve caracterización y se acompaña las fotos para cada especie de abeja encontrada.

PALABRAS CLAVE: Melliponas, camu camu, diversidad, Amazonía peruana

\section{THE NATIVE BEES, MELLIPONAS (HYMENOPTERA: MELLIPONIDAE) ASSOCIATED TO CAMU CAMU Myrciaria dubia}

\section{ABSTRACT}

From April 2005 to February 2006 the inflorescences of camu camu were recorded in five communities of the Amazon river's basin, with the objective to get to know the species and the abundance of native bees associated to this vegetable species. Six genders and sixteen species of native bees were registered and Aphis mellifera as an introduced species. The greater richness of species were presented by the genders Trigona with $43.75 \%$ and Melipona with 25\%. The most abundant species were Trigona sp. 1 with $42.40 \%$ and Melipona eburnea with $22.12 \%$. For each bee that was found it is followed by characterization and photos.

KEYWORDS: Melliponas, camu camu, diversity, Peruvian Amazon. 


\section{INTRODUCCIÓN}

Las melliponas son abejas nativas sín aguijon de los trópicos y subtrópicos,que cumplen una función importante en la polinización de las plantas (Aguilar, 2001). En América tropical la polinización por abejas se ha estimado en entre 30 a $50 \%$ de todas las plantas (Biesmeijer, 1997), 38\% en la región amazónica (Kerr, et al., 2001. En el mundo se conoce cerca de 400 especies de melliponas (Michener, 2000), en el Perú se ha registrado más de 140 especies y en los alrededores de Iquitos 35 especies, algunas nuevas para la ciencia (Rasmussen \& Castillo, 2003). El camu camu Myrciaria dubia (H.B.K.) Mc Vaugh es un frutal amazónico que ha despertado gran interés en la industria mundial, debido a que sus frutos tienen un alto contenido de ácido ascórbico $(900-6112 . \mathrm{mg} / 100 \mathrm{~g}$ de pulpa fresca) (Alves, et al., 2002; Yuyama, et al., 2002). Numerosos estudios se han generado sobre la agronomía, plagas, mejoramiento genético, fitoquímica, procesamiento, mercado, de la planta (Villachica, 1996; Rodríguez, et al.; Pinedo et al., 201; Delgado \& Couturier, 2004; Penn, 2006; Mathews, et al., 2011), pero escasamente se conoce sobre la polinización (Peters \& Vásquez, 1986, Maues \& Couturier, 2002). El objetivo del trabajo fue realizar un inventario de las abejas sin aguijo que vistan las inflorescencia de camu camu, para determinar el impacto en la polinización de la planta.

\section{MATERIALES Y MÉTODOS}

El estudio se realizó de abril de 2005 a febrero del 2006, en las comunidades de Chingana (44' $45^{\prime \prime}$ L.S. y $73^{\circ} 37^{\prime} 9^{\prime \prime}$ L.O.), Sapuena ( $4^{\circ} 41^{\prime} 37^{\prime \prime}$ L.S. y 73³5'36" L.O.), Flor de Castaña ( $4^{\circ} 45^{\prime} 49^{\prime \prime}$ L.S. y $73^{\circ} 35^{\prime} 22^{\prime \prime}$ L.O.), Jenaro Herrera (45 $54^{\prime} 17^{\prime \prime}$ L.S. y $73^{\circ}$ 40' 6" L.O.), Nuevo Pumacahua (4³6'9" L.S. y $73^{\circ} 41^{\prime} 47^{\prime \prime}$ L.O) y Yanallpa $\left(4^{\circ} 52^{\prime} 51^{\prime \prime}\right.$ L.S. $73^{\circ} 46^{\prime 2} 22^{\prime \prime}$ L.O.); localizadas en la cuenca baja del río Ucayali, Municipio de Requena, Región-Loreto. Los suelos están sujetos a las inundaciones estacionales de los ríos y pueden permanecer bajo el agua por un periodo de tres a cuatro meses. La temperatura media anual es de $25.9 \pm 2{ }^{\circ} \mathrm{C}$, la precipitación promedio anual es superior a $2,7 \mathrm{~mm}$, con un periodo de escasa lluvia que va de junio a octubre y un período de mucha lluvia que va de noviembre a mayo (Kvist \& Nebel, 2001).

Las parcelas de cultivos de camu camu se encuentran ocupando terrazas de restingas medias, formadas hace aproximadamente de 10 a 20 años, tienen una extensión promedio de 1 hectárea, entre 6 a 8 años de edad. La colecta de abejas se realizó mediante la técnica del barrido de flores, en 64 plantas que se encontraban en periodo de floración. La unidad de muestreo empleada fue una red entomológica de 40 $\mathrm{cm}$ de diámetro y 3 metros de largo, provisto de una tela de Tull de $0.5 \mathrm{~mm}$ de malla estirada. El material biológico colectado se encuentra depositado en las colecciones entomológicas del Instituto de Investigaciones de la Amazonía Peruana.

\section{RESULTADOS Y DISCUSIÓN}

Durante el estudio se colectó 434 individuos distribuidos en 6 géneros y 16 especies de abejas nativas y Aphis mellifera como especie introducida. La mayor riqueza de especies presenta el género Trigona con $43.75 \%$ y Melipona con $25 \%$ (Tabla. 1, Figura 2). Las especies más abundantes fueron Trigona sp. 1 con $42.40 \%$ y Melipona eburnea con $22.12 \%$, las menos abundantes fueron Trigona pallens, Cephalotrigona sp. y Tetragona clavipes con $0.23 \%$ cada una. Si consideramos la especie introducida Aphis melliphera, esta se encuentra en el segundo lugar con el $32.3 \%$ del total de individuos registradas.

En Perú, en poblaciones naturales de Myrciaria dubia, fueron encontradas dos especies de meliponas (Peters \& Vásquez, 1986) y en Brasil, en una plantación experimental en la región de Belem do Para, fue encontrada siete especies (Maues \& Couturier, 2002). La mayor diversidad de especies de meliponas encontradas en la zona baja del rio Ucayali se puede atribuir a la edad de las plantas $(08$ años) en comparación con la plantación experimental en Belem do Para (3 años), que es la edad de inicio de floración de la especie. Otro factor sería la extensión de las parcelas, que en muchos casos son contiguas que van formando grandes áreas. La mayor abundancia de individuos se concentra en dos especies, siendo diferentes para cada región; en Belem do Para fueron Nannotrigona punctata y Trigona pallens (Maues \& Couturier, 2002), mientras que en el bajo Ucayali fueron Trigona sp.1 y Melipona eburnea. Una flor puede ser visitado por diferentes especies al mismo tiempo, pero la especie introducida Aphis melifera ejerce un comportamiento agresivo frente a las especies nativas. Este comportamiento, sumado a la abundancia y frecuencia de Aphis melifera en las plantaciones de camu camu, puede haber influenciado en la presencia de una menor diversidad de especies colectadas.

Caracterización morfológica de las especies de abejas nativas encontradas en inflorescencia del camu camu

\section{Melipona eburnea Friese, 1900}

Esta especie es conocida en nuestra región como "abeja pacucho" (en quechua pacucho significa 
rubio), "ronsapilla" o "abeja mansa". Son de color castaño claro, con abundantes pelos plumosos y semi erectos de color marrón en todo el cuerpo. Alas anteriores relativamente más pequeñas que el abdomen con ángulo submarginal bastante agudo y pterostigma casi lineal. Margen distal de las alas posteriores con 13 hámulis. Superficie interna de la tibia posterior sin faja marginal, área con quirotriquias densa extendiéndose hasta el borde de la tibia posterior, fajas abdominales enteras y gruesas de color blanco marfil (Figura 1A).

\section{Melipona grandis, Guerin, 1844}

Son abejas de color negro, llegan a medir hasta 15 $\mathrm{mm}$ de longitud, cuerpo con abundantes pelos largos, muy finos y plumosos de color blanco. Alas anteriores más cortas que el abdomen con ángulo submarginal agudo y pterostigma lineal. Margen distal de las alas posteriores con 15 hámulis. Superficie interna de la tibia posterior sin faja marginal, área con quirotriquias densa y extendiéndose hasta el borde de la tibia, extremo distal posterior de la tibia posterior en ángulo muy agudo, partes laterales del mesotórax con un mechón de color marrón, pelos dorados en el basitarso, blanquecinos y marrones en el resto del cuerpo, último segmento abdominal terminando en punta o ángulo muy pronunciado, con fajas abdominales de color amarillo claro, sólo con la primera faja entera o continua (Figura 1A).

\section{Melipona illota Cockerell, 1919}

Abejas de color negro grisáceo con abundantes pelos plumosos de color marrón y cerdas negras en todo el cuerpo. Alas anteriores relativamente pequeñas en comparación al abdomen, ángulo submarginal agudo o casi recto y pterostigma lineal. Margen distal de las alas posteriores con 13 hámulis. Superficie interna de la tibia posterior sin faja marginal, área con quirotriquias no tan densa como en Melipona grandis y extendiéndose hasta el borde de la tibia, extremo distal posterior de la tibia posterior terminando en ángulo no muy pronunciado, abdomen redondeado con fajas abdominales marrones enteras o continuas (Figura 1A).

\section{Melipona fuliginosa Lepeletier, 1836}

Abejas grandes y robustas, miden de $12-15 \mathrm{~mm}$ de longitud, son enteramente negras brillantes. Alas anteriores con ángulo submarginal casi recto y pterostigma casi lineal. Margen distal de las alas posteriores con 14 hámulis. Presenta abundantes pelos largos, erectos y plumosos de color negro en todo el cuerpo. Superficie interna de la tibia posterior sin faja marginal, área con quirotriquias no tan densa y extendiéndose hasta el borde de la tibia, extremo distal posterior de la tibia posterior terminando en ángulo no muy pronunciado abdomen sólo con la primera faja abdominal entera y visible de color marrón (Figura 1A).

\section{Trigona amalthea (Olivier, 1789)}

Abejas más o menos robustas, enteramente negras. Alas anteriores de color negro muy desarrolladas, más largas que el abdomen, ángulo submarginal abierto y pterostigma poco desarrollado. Margen distal de las alas posteriores con 5 hámulis. Superficie interna de la tibia posterior con faja marginal glabra y fuertemente deprimida, área con quirotriquias levantada formando un amplio surco, extremo distal posterior de la tibia posterior redondeado, borde posterior de la tibia posterior con abundantes pelos largos y fuertemente plumosos entre las cerdas simples, basitarsos posteriores con área sedosa basal no tan densa, abdomen redondeado, no se diferencian las fajas abdominales (Figura 1A).

\section{Trigona pallens (Olivier, 1789)}

Abejas pequeñas totalmente de color castaño claro, con abundantes pelos dorados en todo el cuerpo. Alas anteriores relativamente más largas que el abdomen, ángulo submarginal semi abierto $\mathrm{y}$ pterostigma más o menos desarrollado. Margen distal de las alas posteriores con 5 hámulis. Superficie interna de la tibia posterior con faja marginal glabra y fuertemente deprimida, área con quirotriquias levantada formando un surco en el centro de la superficie interna de la tibia posterior, margen posterior de la tibia posterior con abundantes pelos largos y plumosos, extremo distal posterior de la tibia posterior redondeado, borde posterior de la tibia posterior basitarso posterior con área sedosa basal, abdomen estrecho y transparente, no se diferencian las fajas abdominales (Figura 1A).

\section{Trigona sp. 1 Jurine, 1807}

Abejas pequeñas, enteramente negras brillantes. Alas anteriores blanquecinas ligeramente más largas que el abdomen, ángulo submarginal semi abierto y pterostigma más o menos desarrollado. Margen distal de las alas posteriores con 5 hámulis. Presenta abundantes pelos erectos de color negro en el tórax. Superficie interna de la tibia posterior con faja marginal glabra más o menos amplia y ligeramente deprimida, área con quirotriquias formando un surco amplio a lo largo de los $2 / 3$ basales de la margen posterior de la tibia, extremo distal posterior de la tibia posterior redondeado, borde posterior de la tibia posterior con abundantes pelos fuertemente plumosos entre las cerdas simples, abundantes pelos 
plumosos agrupados en el extremo anterior de la superficie externa de la tibia posterior, basitarso posterior con área sedosa basal densa, abdomen muy delgado y alargado, fajas abdominales enteras de color negro opaco (Figura 1B).

\section{Trigona sp. 2 Jurine, 1807}

Son abejas medianas de color marrón. Poseen abundantes pelos dorados en todo el cuerpo. Alas anteriores relativamente más grandes que el abdomen, ángulo submarginal semmi abierto y pterostigma más o menos desarrollado. Margen distal de las alas posteriores con 5 hámulis. Superficie interna de la tibia posterior con faja marginal glabra y fuertemente deprimida, área con quirotriquias levantada formando un surco amplio a lo largo de la margen de la tibia, extremo distal de la tibia posterior redondeado, borde posterior de la tibia posterior con abundantes pelos largos y fuertemente plumosos, extremo anterior de la superficie externa de la tibia posterior sin pelos, tibias posteriores marrones oscuras con área de color castaño claro en la superficie externa del fémur y tibia posterior, basitarsos posteriores con área sedosa basal densa, abdomen con fajas abdominales gruesas y de color marrón oscuro (Figura 1B).

\section{Trigona sp. 3 Jurine, 1807}

Abejas grandes, con la cabeza, el tórax y los 2 primeros pares de patas de color negro, abdomen y patas posteriores de color marrón. Alas anteriores más largas que el abdomen, ángulo submarginal abierto y pterostigma más o memos desarrollado. Margen distal de las alas posteriores con 6 hámulis. Presenta abundantes pelos blanquecinos, plumosos y cortos, cerdas simples y semi erectas en todo el cuerpo. Superficie interna de la tibia posterior con faja marginal glabra amplia, fuertemente deprimida y ligeramente rebajada en relación al área con quirotriquias. Área con quirotriquias formando un surco a lo largo de los $2 / 3$ basales de la margen posterior de la tibia, extremo distal posterior de la tibia posterior redondeado, borde posterior de la tibia posterior con abundantes pelos largos y fuertemente plumosos entre las cerdas simples, sin pelos en el extremo anterior de la superficie externa de la tibia posterior, basitarsos posteriores con área sedosa basal densa, abdomen alargado, abdomen con fajas abdominales gruesas más o menos definidas de color marrón oscuro (Figura 1B).

\section{Trigona sp. 4 Jurine, 1807}

Abeja pequeña y robusta de color negro, abdomen corto y triangular. Alas anteriores excediendo bastante en largo al abdomen, ángulo submarginal abierto y pterostigma más o menos desarrollado.
Margen distal de las alas posteriores con 5 hámulis. Presenta escasos pelos cortos y semi erectos. Superficie interna de la tibia posterior con faja marginal glabra amplia, fuertemente deprimida y ligeramente rebajada en relación al área con quirotriquias. Área con quirotriquias formando un surco a lo largo de los $2 / 3$ basales, extremo distal posterior de la tibia posterior redondeado, borde posterior de la tibia posterior con abundantes pelos fuertemente plumosos entre las cerdas simples, sin pelos en el extremo anterior de la superficie externa de la tibia posterior, basitarso posterior con área sedosa basal densa, abdomen con segmentos abdominales reducidos y fajas abdominales no visibles (Figura 1B).

\section{Trigona sp. 5 Jurine, 1807}

Abejas pequeñas, con el tórax cabeza y dos primeros par de patas de color negro, abdomen corto y ligeramente delgado de color marrón al igual que las patas posteriores. Alas anteriores de color marrón excediendo bastante en largo al abdomen, ángulo submarginal semi abierto y pterostigma más o menos desarrollados. Margen distal de las alas posteriores con 5 hámulis. Presenta escasos pelos cortos y semi erectos. Superficie interna de la tibia posterior con faja marginal glabra amplia, fuertemente deprimida y ligeramente rebajada en relación al área con quirotriquias. Área con quirotriquias formando un surco a lo largo de los $2 / 3$ basales, extremo distal posterior de la tibia posterior redondeado, borde posterior de la tibia posterior con abundantes pelos fuertemente plumosos entre las cerdas simples, sin pelos en el extremo anterior de la superficie externa de la tibia posterior, basitarso posterior con área sedosa basal densa, abdomen con fajas abdominales no visibles (Figura 1B).

\section{Partamona sp. Schwarz, 1938}

Abejas pequeñas de color negro. Alas anteriores más largas que el abdomen, ángulo submarginal semi abierto y pterostigma muy desarrollado. Margen distal de las alas posteriores con 5 hámulis. Integumento liso y brillante, escutelo relativamente corto pelos muy cortos, finos y semi erectos en todo el cuerpo, espacio malar igual al diámetro del flagelo. Tibia posterior muy ensanchada con faja marginal glabra muy estrecha, área con quirotriquias extendiéndose casi hasta el borde de la superficie de la de la tibia, extremo distal de la tibia posterior terminando en ángulo no muy pronunciado, margen posterior de la tibia posterior con pelos simples, corbícula amplia ocupando casi toda la superficie externa de la tibia, abdomen achatado casi de igual longitud que el tórax, no se distinguen las fajas abdominales (Figura 1C). 
13. Scaptotrigona sp.n. nigrohirta. Moure, 1968

Abejas pequeñas y robustas de color marrón. Alas anteriores con pterostigma más o menos desarrollado y ángulo submarginal semi abierto. Margen distal de las alas posteriores con 7 hámulis. Espacio malar excediendo en diámetro al flagelo. Parte anterior del tórax de color negro, base del escutelo con una entrada en forma de "V". Superficie interna de la tibia sin faja marginal glabra, área con quirotriquias extendiéndose hasta el borde de la tibia posterior, extremo distal de la tibia terminando en ángulo no muy pronunciado, corbícula amplia muy desarrollada ocupando toda la superficie externa de la tibia, borde posterior de la tibia posterior con pelos simples, parte anterior del abdomen de color negro, con fajas abdominales gruesas y enteras de color marrón (Figura 1C).

\section{Cephalotrigona sp. Schwarz, 1940}

Son abejas relativamente grandes y robustas de color negro opaco. Alas anteriores de igual tamaño que el abdomen, con ángulo submarginal abierto $\mathrm{y}$ pterostigma más o menos desarrollado. Margen distal de las alas posteriores con 10 hámulis. Presentan pelos blanquecinos cortos y finamente plumosos en todo el cuerpo, espacio malar más largo que el diámetro del flagelo; diente basal de la mandíbula bastante desarrollada. Tibia posterior muy ensanchada con faja marginal glabra amplia y fuertemente deprimida, área con quirotriquias ligeramente levantada no formando surco amplio a lo largo de la margen de la tibia, extremo distal de la tibia redondeado, borde posterior de la tibia posterior con pocos pelos cortos y simples entre cerdas simples, corbícula amplia ocupando casi toda la superficie externa de la tibia, abdomen con fajas abdominales muy finas y enteras de color marrón (Figura 1C).

15. Tetragona clavipes (Fabricius, 1804)

Abejas pequeñas de color negro. Alas anteriores relativamente más pequeñas que el abdomen, con ángulo submarginal semi abierto y pterostigma más o menos desarrollado. Margen distal de las alas posteriores con 5 hámulis. Presenta abundantes pelos dorados en todo el cuerpo, patas marrones, excepto la tibia y basitarso posterior que son de color negro. Superficie interna de la tibia posterior con faja marginal glabra amplia y fuertemente deprimida, borde posterior de las tibias posteriores con pocos pelos fuertemente plumosos entre cerdas simples, tibia media con espolón, superficie interna del basitarso posterior muy reducido en comparación a la tibia posterior apenas con cerdas simples y sin área sedosa basal, extremo distal de la tibia posterior redondeado, triángulo propodeal glabro, abdomen estrecho con fajas abdominales gruesas, enteras y de color amarillo (Figura 1C).

\section{Tetragona sp. $2(\mathrm{Otto}, 1823)$}

Abejas de tamaño medio, de color marrón oscuro. Alas anteriores muy desarrolladas, más largas que el abdomen, con ángulo submarginal abierto y pterostigma más o menos desarrollado. Margen distal de las alas posteriores con 6 hámulis. Presenta escasos pelos cortos y erectos de color dorado, mandíbula con tres dientes conspicuos, patas marrones, superficie interna de la tibia posterior con faja marginal glabra amplia y rebajada en relación al área con quirotriquias, borde posterior de las tibias posteriores con pelos fuertemente plumosos entre las cerdas simples. Superficie interna del basitarso posterior bastante pequeño en comparación con la tibia posterior y apenas con cerdas simples y sin área sedosa basal, extremo distal de la tibia posterior redondeado, tibia media con espolón, triángulo propodeal prácticamente glabro, abdomen ligeramente grueso con fajas abdominales gruesas, definidas y de color marrón más oscuro que el resto del abdomen (Figura 1C). 
Tabla 1. Diversidad de abejas nativas encontradas en inflorescencia de camu camu.

\begin{tabular}{|c|c|c|}
\hline Tribu & Género & Especie \\
\hline & Melipona & Melipona eburnea Friese, 1900 \\
\hline \multirow[t]{8}{*}{ Meliponini } & & Melipona grandis, Guerin, 1844 \\
\hline & & Melipona illota Cockerell, 1919 \\
\hline & & Melipona fuliginosa Lepeletier, 1836 \\
\hline & Trigona & Trigona sp. 1 Jurine, 1807 \\
\hline & & Trigona amalthea (Olivier, 1789) \\
\hline & & Trigona sp 2 Jurine, 1807 \\
\hline & & Trigona sp 3 Jurine, 1807 \\
\hline & & Trigona sp 4 Jurine, 1807 \\
\hline \multirow[t]{7}{*}{ Trigonini } & & Trigona sp 5 Jurine, 1807 \\
\hline & & Trigona pallens (Fabricius, 1793) \\
\hline & Partamona & Partamona sp. Schwarz, 1938 \\
\hline & Scaptotrigona & Scaptotrigona sp.n.nigrohirta Moure, 1968 \\
\hline & Tetragona & Tetragona sp 2 (0tto, 1823) \\
\hline & & Tetragona clavipes (Fabricius, 1804) \\
\hline & Cephalotrigona & Cephalotrigona sp. Schwarz, 1940 \\
\hline
\end{tabular}

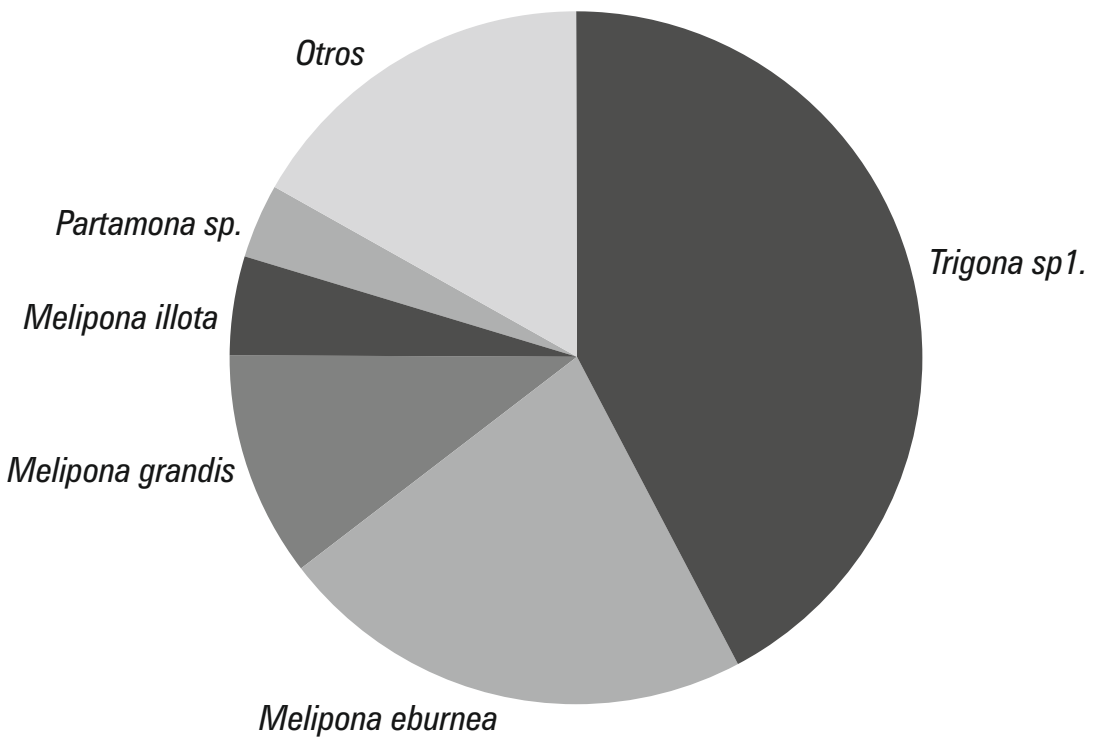

Figura 2. Abundancia relativa de las principales especies de abejas nativas encontradas en inflorescencia de camu camu. 


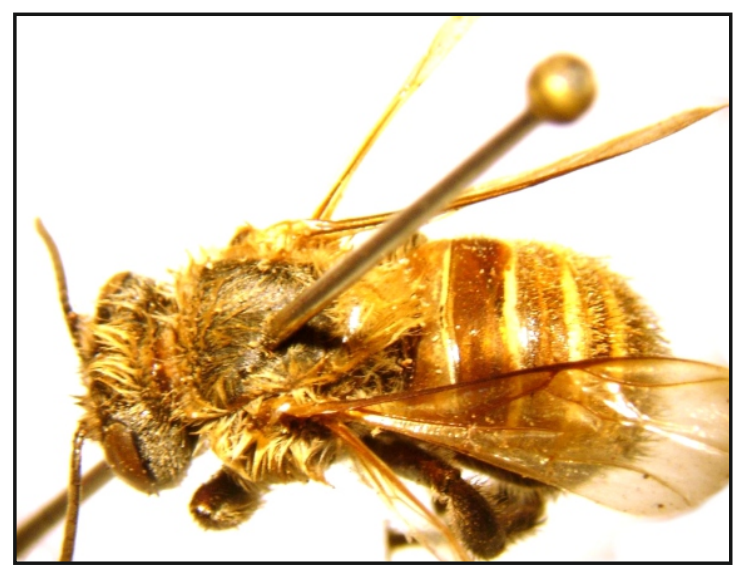

Melipona ebúrnea

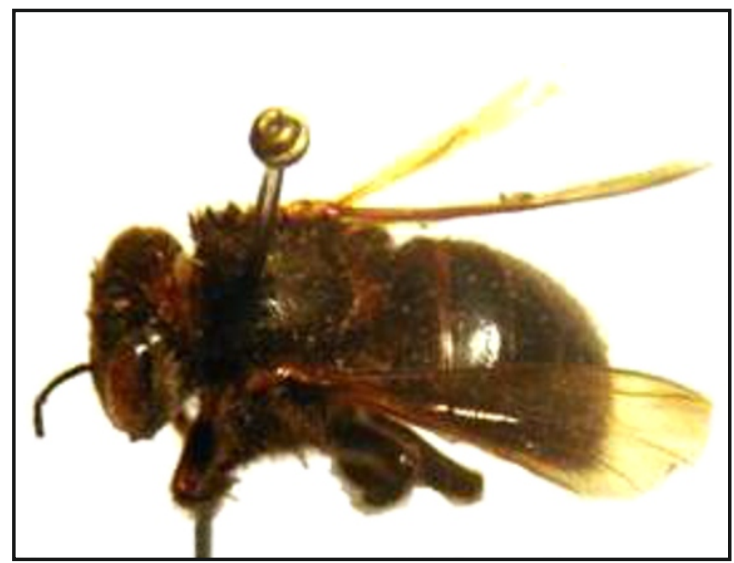

Melipona illota

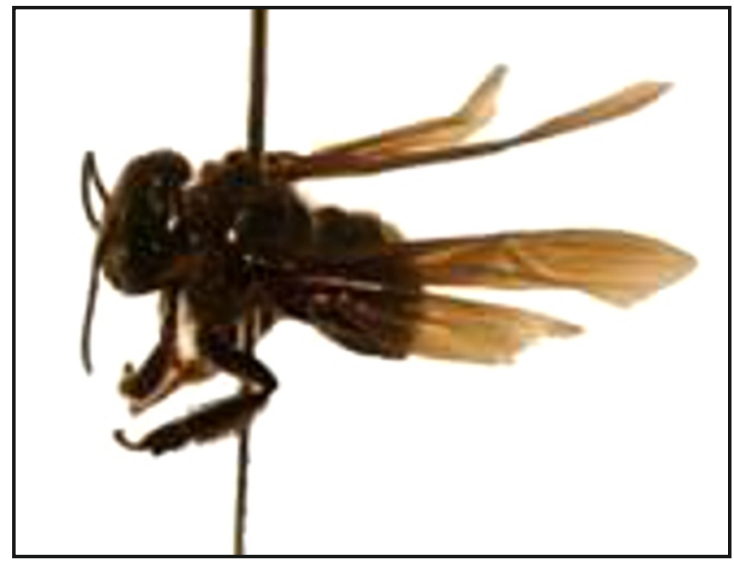

Trigona amalthea

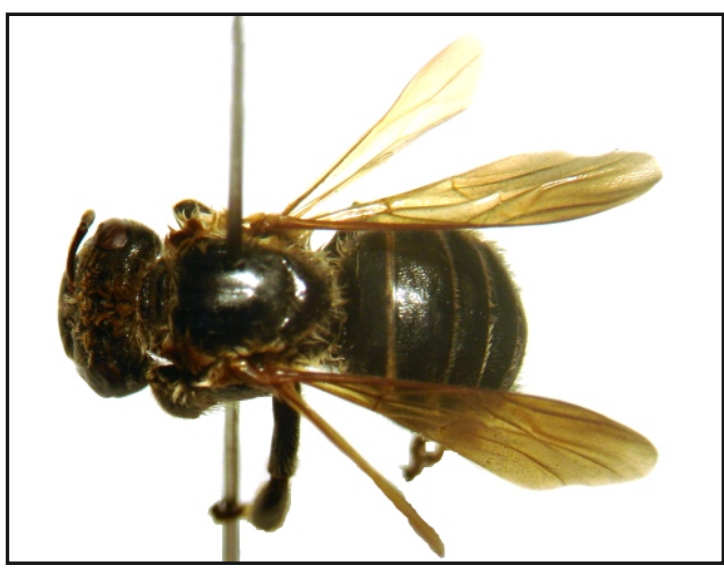

Melipona grandis

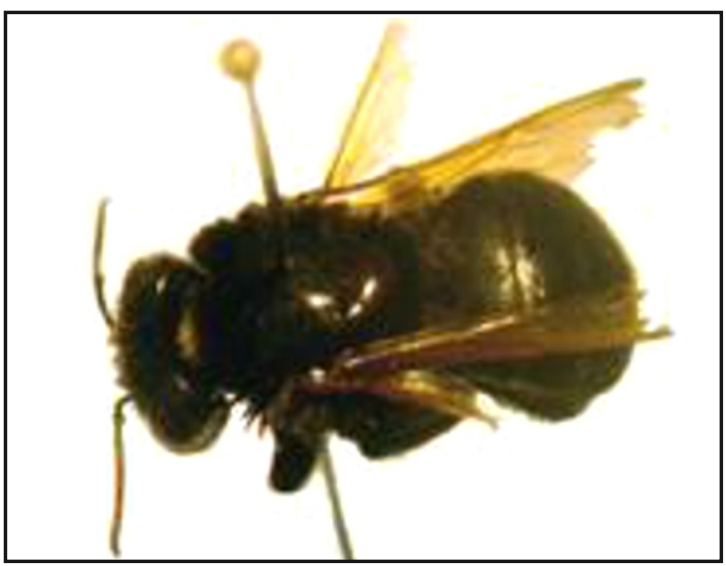

Melipona fuliginosa

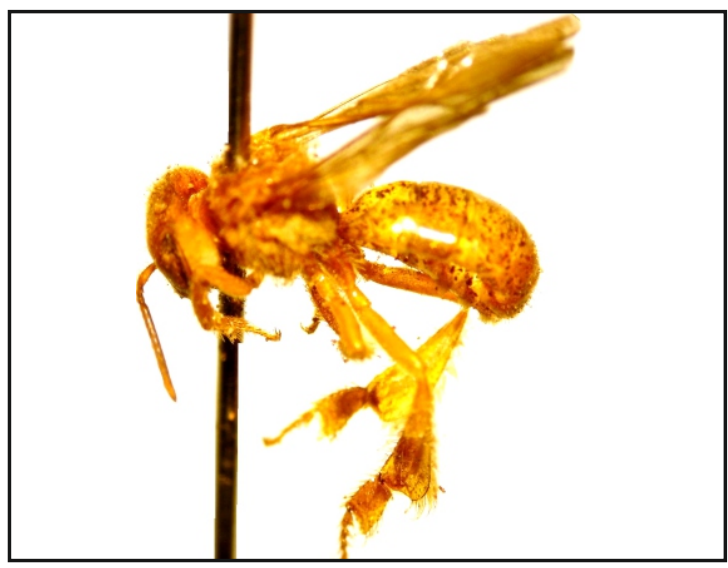

Trigona pallens

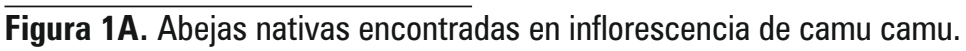




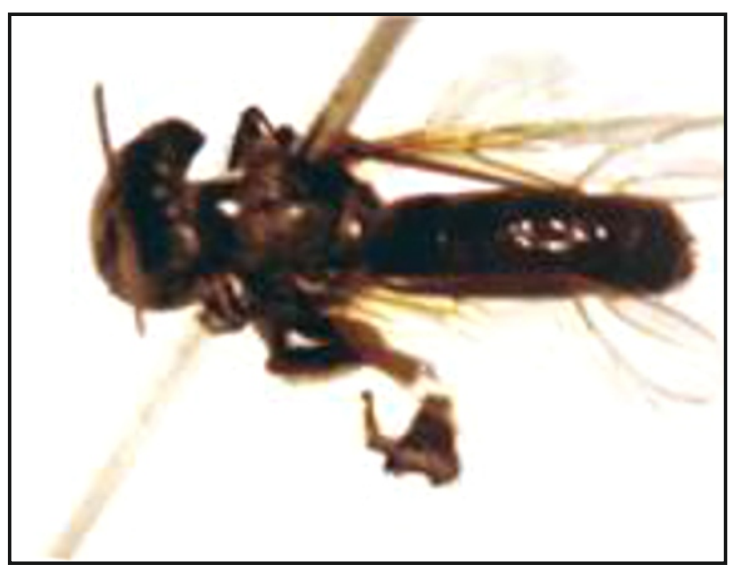

Trigona sp. 1

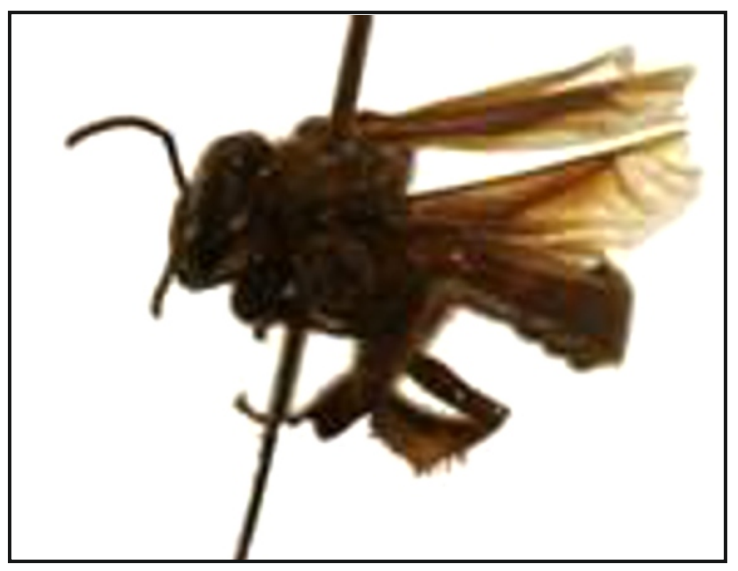

Trigona sp. 3

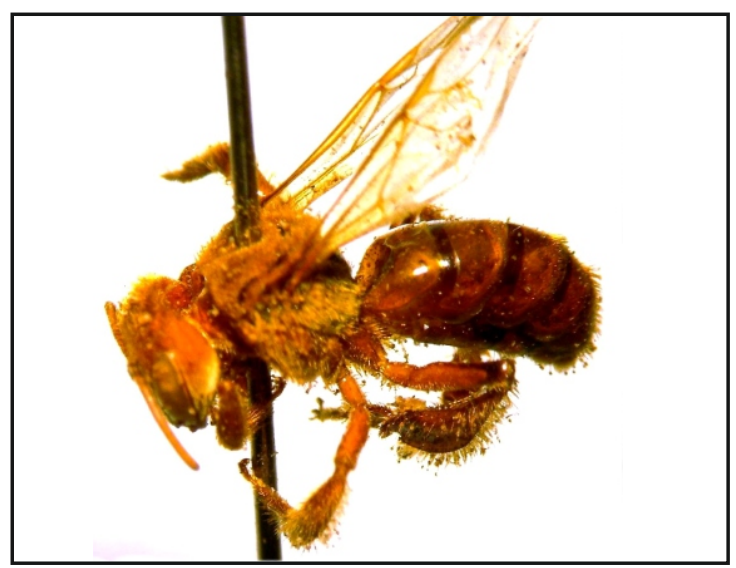

Trigona sp. 2

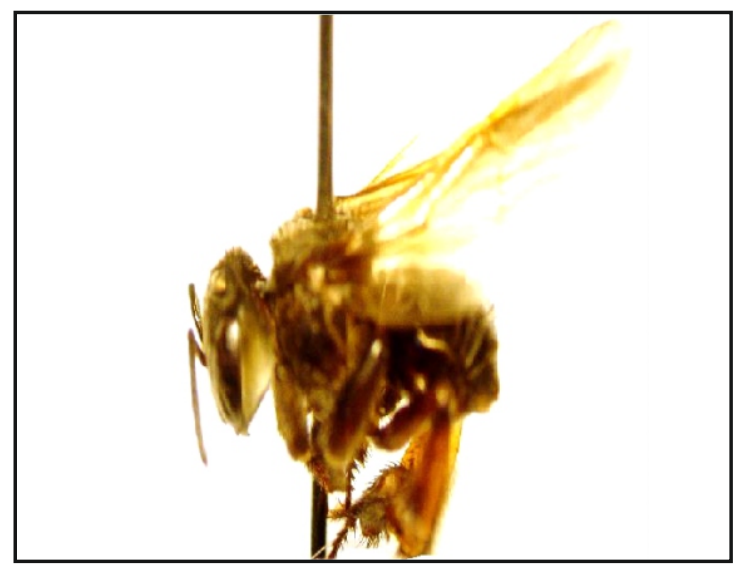

Trigona sp. 4

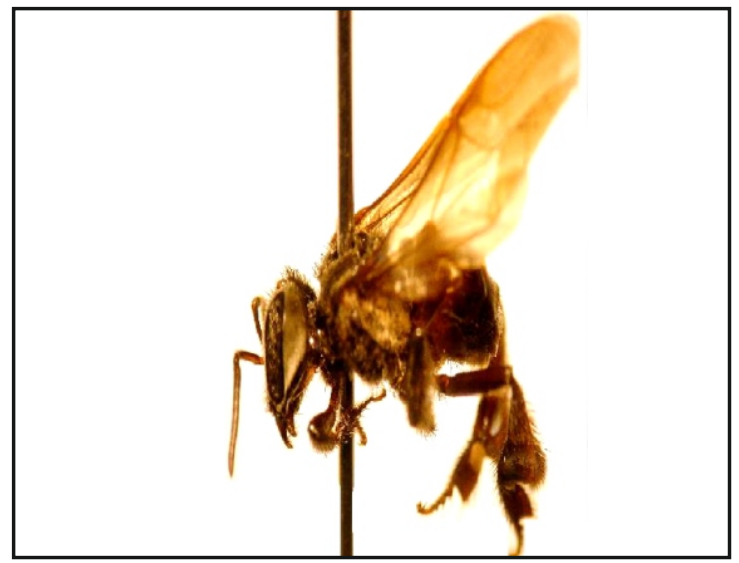

Trigona sp. 5

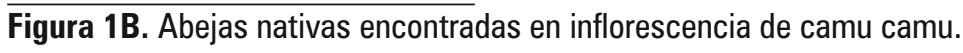




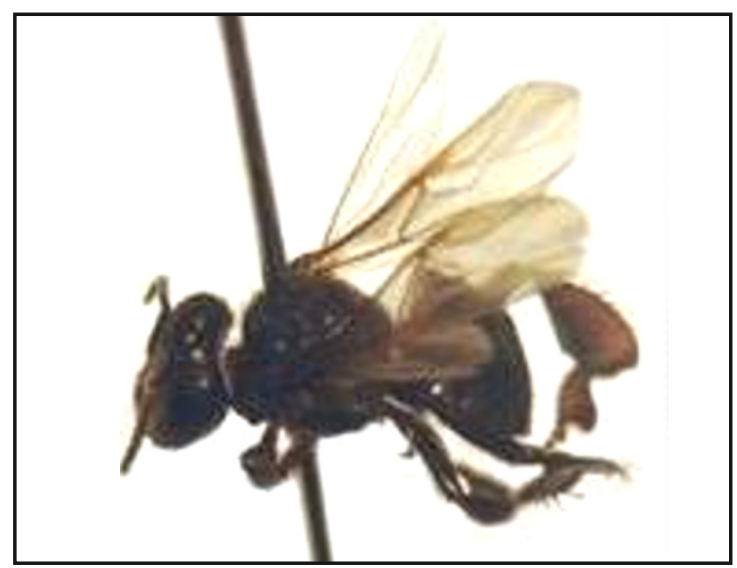

Partamona sp.

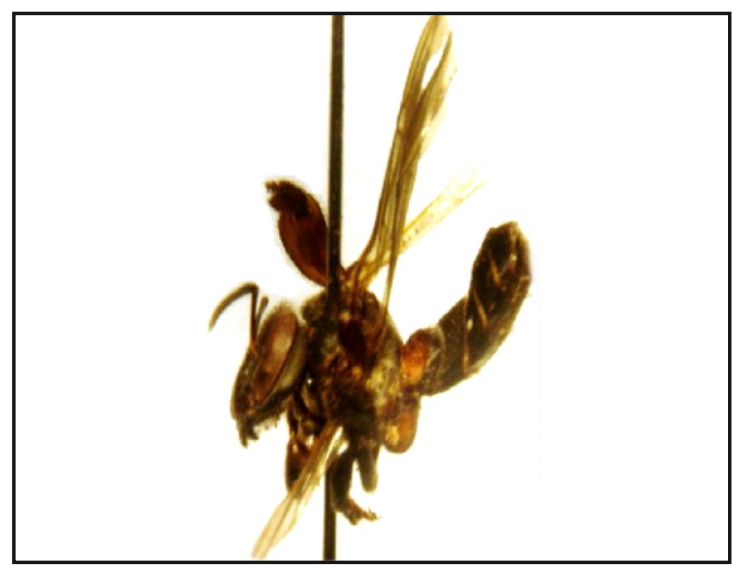

Cephalotrigona sp.

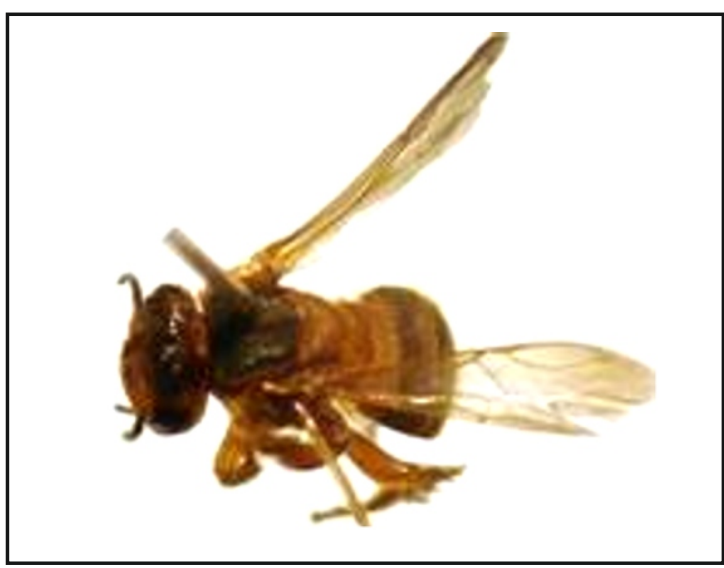

Scaptotrigona sp.n nigrohirta

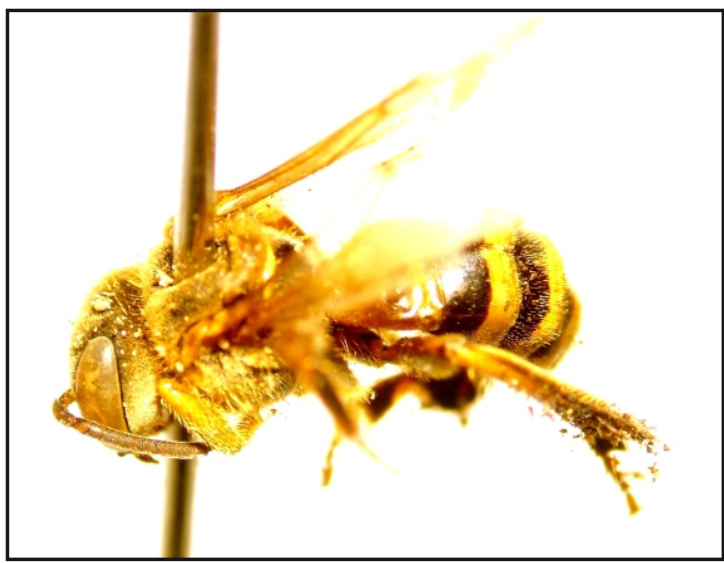

Tetragona clavipes

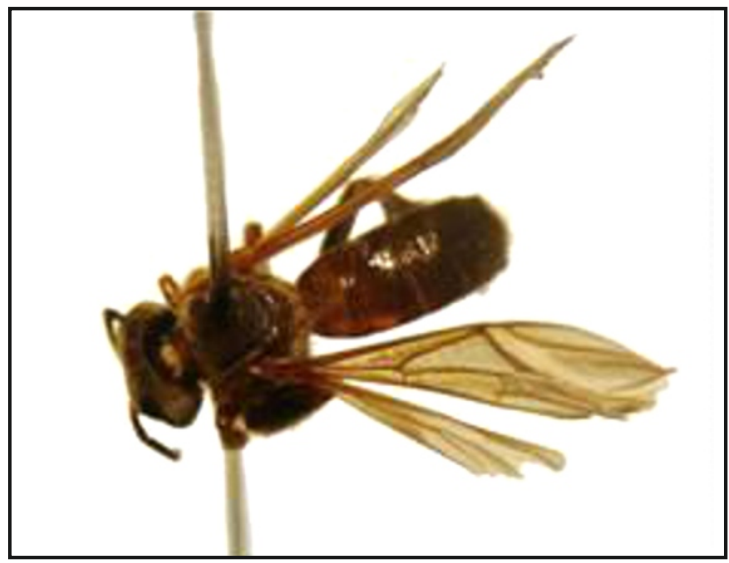

Tetragona sp. 2

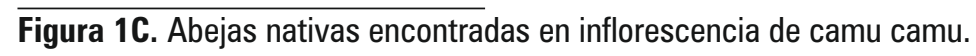




\section{AGRADECIMIENTO}

El trabajo financiado parcialmente por INCAGRO/ conducido por el Instituto de Investigaciones de la Amazonía Peruana. Agradecemos al Dr. Claus Rasmussen por la identificación de las especies, a Jeronimo Vega por el apoyo en el trabajo de Laboratorio y a todos los agricultores de las comunidades de Sapuena, Chingana, Nuevo Pumacahua y Flor de Castaña por el apoyo y facilidades en sus parcelas.

\section{BIBLIOGRAFIA CITADA}

Aguilar, M. I. 2001. Como manejar abejas sin aguijón (Apidae: Meliponinae) en sistemas agroforestales. Revista Agroforestería en las Américas, 8(31): 50-55

Alves, R.E.; Figueiras, H.A.C.; Moura, C.F.H.; Araujo, N.C.C.; Almeida, A.S. 2002. Camu camu (Myrciaria dubia Mc Vaugh): A rich natural source of vitamin $C$. Proc. Interamer. Soc. Trop. Hort., 46:11-13.

Biesmeijer, J. 1997. Abejas sin aguijón. Elinkwiijk BV. Utrech, The Netherlands. 77 pag.

Delgado, C.; Couturier, G. 2004. Manejo de insectos plagas en la Amazonía: Su aplicación en camu camu. IIAP-IQUITOS / IRD-FRANCIA, Lima 2004. 147 pág.

Kerr, W.E.; Carvalho C.A.; Silva, A.C.; Assis, M.G.P. 2001. Aspectos pouco mencionados da biodiversidade Amazônica. Parcerias estratégicas (Brasília), 12:20-41.

Kvist, L.P.; Nebel, G. 2001. A review of Peruvian flood plain forests: ecosystems, inhabitants and resource use. Forest Ecology and Management, 150:3-26.

Mathews, J.P.; Bardales, R.; Delgado, C.; Pinedo M. 2011. Enraizamento de estacas de camu-camu com diferentes diâmetros sob diferentes concentrações do ácido indolbutírico. Anais do I workshop Agricultura no Trópico Úmido., Manaus-Brasil.

Maues, M.M.; Couturier, G. 2002. Biología Floral y Fenología Reproductiva del camu camu. Revista Brasilera de Botánica, 25(4): 1-13.

Michener, C.D. 2000. The bees of the world. Baltimore, Johns Hopkins University Press.

Penn, J.W. 2006. The cultivation of camu camu (Myrciaria dubia): a tree planting programme in the Peruvian Amazon. Forests, trees and livelihoods, 16:85-101.

Peters, C.M.; Vasquez, A. 1986. Estudios ecológicos de camu camu (Myrciaria dubia). Producción de frutos en poblaciones naturales. Acta Amazónica, 16(17): 161-174.

Pinedo, M.; Delgado, C.; Iman, S.; Farroñay, R.; Villacrés, J. 2011. Camu-camu Myrciaria dubia ): aportes a su aprovechamiento sostenible en la Amazonía peruana, IIAP- Perú. 135pp.

Rasmussen, C.; Castillo, P.S. 2003. Estudio preliminar de la meliponicultura o apicultura silvestre en el Perú (Hymenoptera: Apidae, Meliponini). Re. Per, Ent., 43:159-164.

Rodríguez, R.B.; De Menezes, H.C.; Cabral, L.M.C.; Dornier, M.; Reynes, M. 2001. An amazonian fruit with high potential as a natural source of vitamin C: the camu-camu (Myrciaria dubia). Fruits, 56(5):345-354.

Villachica, H. 1996. El cultivo de camu camu (Myrciaria dubia H. B. K. Mc Vaugh) en la Amazonía Peruana. Tratado de Cooperación Amazónica, Lima, Perú.

Yuyama, K.; Aguiar, J.; Yuyama, K.O.L. 2002. Camu-camu: Um fruto fantástico como fonte de vitamina C. Acta Amazónica, 32(1):170-173. 